\title{
Effects of physical and chemical surface roughness on the brightness of electron beams from photocathodes
}

\author{
G. S. Gevorkyan, S. Karkare, and S. Emamian \\ Lawrence Berkeley National Laboratory, Berkeley, California 94720, USA \\ I. V. Bazarov \\ Cornell University, Ithaca, New York 14853, USA \\ H. A. Padmore \\ Lawrence Berkeley National Laboratory, Berkeley, California 94720, USA
}

\begin{abstract}
(Received 6 July 2018; published 14 September 2018; corrected 1 November 2018 and 16 April 2020)
\end{abstract}
\begin{abstract}
The performance of free electron laser x-ray light sources, and systems for ultrafast electron diffraction and ultrafast electron microscopy, is limited by the brightness of the electron sources used. The intrinsic emittance, or equivalently, the mean transverse energy (MTE) of electrons emitted from the photocathode determines the maximum possible brightness in such systems. With ongoing improvements in photocathode design and synthesis, we are now at a point where the physical and chemical surface roughness of the cathode can become a limiting factor. Here we show how measurements of the spatially dependent variations in height and surface potential can be used to compute the electron beam mean transverse energy (MTE), one of the key determining factors in evaluation of brightness.
\end{abstract}

DOI: 10.1103/PhysRevAccelBeams.21.093401

\section{INTRODUCTION}

Photoinjectors can provide high-brightness electron beams that are suitable for use in a wide variety of applications, from free electron lasers [1] to ultrafast electron diffraction and microscopy [2] setups. A key figure of merit for these systems is the 4D beam brightness. The maximum possible $4 \mathrm{D}$ brightness $\left(B_{4 \mathrm{D}}\right)$ from a photoinjector is proportional to the accelerating field $\left(E_{z}\right)$ at the photocathode and inversely proportional to the mean transverse energy (MTE) of the photoexcited electron beam at the cathode surface [3], $B_{4 D} \propto E_{z} /(\mathrm{MTE})$. The MTE can be related to the intrinsic cathode emittance $(\epsilon)$ via the relation $\epsilon=\sigma \sqrt{\mathrm{MTE} /\left(m_{0} c^{2}\right)}$, where, $\sigma$ is the rms laser spot size on the cathode, $m_{0}$ is the electron rest mass and $c$ is the speed of light.

For cathodes with disordered surfaces MTE is roughly equal to $E_{\text {ex }} / \alpha$, where $E_{\text {ex }}$ is the excess energy (the energy difference between the photon energy and the work function) and $\alpha$ is generally equal to 3 for ideal metals, but can vary because of several factors such as the band structure, electron scattering during emission etc. [4]. As the excess energy approaches the thermal energy, the

Published by the American Physical Society under the terms of the Creative Commons Attribution 4.0 International license. Further distribution of this work must maintain attribution to the author(s) and the published article's title, journal citation, and DOI.
MTE is limited to $k_{B} T$ by the thermal energy [5]. This has been experimentally demonstrated and an MTE equal to $25 \mathrm{meV}\left(k_{B} T\right.$ at room temperature) from thin Sb films had been measured [5]. The penalty that has to be paid for operating at excess energies comparable to $k_{B} T$ is in the quantum efficiency $(\mathrm{QE})$ of the cathode.

For a simple free electron metal, where most of the scattered electrons do not get emitted, the QE is quadratically related to the excess energy; for example, reducing from an excess energy typical of today's photoinjectors or $0.5 \mathrm{eV}$ to room temperature $k_{B} T$, would result in a factor of 400 lower QE. In principle, the deficit in the bunch charge due to a lower QE can be compensated for by increasing the laser pulse energy, up to the point where direct electron heating in the solid becomes significant [6]. Another way is to use high QE semiconductor cathodes like alkaliantimonides or III-V semiconductors activated to negative electron affinity. Such materials have a large QE as a large fraction of scattered electrons do get emitted $[7,8]$.

MTE smaller than $22 \mathrm{meV}$ along with a $\mathrm{QE}$ as high as $7 \times 10^{-5}$ has been measured from alkali-antimonide based cathodes at liquid nitrogen temperatures close to the photoemission threshold [9]. In this case, the MTE did not reach $8 \mathrm{meV}$ with $k_{B} T$ at liquid nitrogen temperatures. The MTE was limited to a much higher value, making it certain that several other effects can become dominant as we try to generate electron beams with smaller MTE. The main two, which we examine here, are effects due to the physical roughness of the surface of the cathode, and due to 
local transverse gradients in the surface potential caused by changes in work function of a metal cathode, or by the changes in the gap and electron affinity of a semiconductor cathode.

In a photoinjector, the photocathode is immersed in a strong longitudinal electric field gradient typically in the range $5-100 \mathrm{MV} / \mathrm{m}$. If a small area of the surface is tilted due to local roughness, this will produce a transverse electric field gradient which will increase the MTE correlated to the magnitude of the applied electric field. The effect will depend on the local tilt of the surface and so even small surface height variations, if accompanied by a small periodicity, can produce large effects. In addition, most photocathodes used today are polycrystalline, and these photocathodes will exhibit changes in local work function depending on their chemical composition and the local orientation of the crystallite. This effect is particularly significant in multielement photocathodes, such as the alkali-antimonides, due to local changes in stoichiometry [10].

Due to their high QE even at photon energies close to threshold, alkali-antimonides are one of the few materials capable of delivering low emittance electron beams with large bunch charges. Although codeposition methods have reduced the physical roughness and the extent of local changes in potential in alkali-antimonides [11], it is common to find height variations of a few $\mathrm{nm}$ and chemical potential amplitudes of $0.1 \mathrm{~V}$ over length scales of 50-200 nm.

These variations can be measured quite accurately using atomic force microscopy (AFM) and Kelvin probe force microscopy (KPFM). As we advance from an MTE of several hundred $\mathrm{meV}$ to the few $\mathrm{meV}$ range, we will need to find ways to control both forms of local variation. The aim of this work is to demonstrate a method to use measured cathode surface roughness and potential variations to predict the ultimate MTE limited by the surface nonuniformities that can be achieved for a defined accelerating gradient for the particular cathode.

Previously, several authors have calculated the effects of physical roughness on MTE [12-14] using various approaches. Here we discuss the works that are most relevant to our approach. Bradley developed an analytic expression for the MTE from a 1-D sinusoidal surface variation for the case where the amplitude of the variation was much smaller than the period [15]. This approximation to the electric field was used to estimate increase in emittance due to a sinusoidal physical roughness accurately [16]. Zhang and Tang extended this formalism to 2-D surfaces with realistic surface roughness by expanding the surface in terms of its Fourier coefficients [17]. Gorlov used a more precise, combined analytic numerical method of the 3-D field calculation close to a realistic 2-D surface [18]. This method has been used to obtain the MTE increase from the physical roughness on realistic photocathode surfaces measured using an AFM [19,20]. Despite the varying complexity of these studies, all of them conclude that the MTE increase due to the physical roughness is proportional to the accelerating electric field at the cathode surface.

More recently Karkare and Bazarov have investigated the effects of chemical roughness or varying surface potentials on the MTE from surfaces with no physical roughness [21]. They showed that surface potential variations as small as $100 \mathrm{mV}$ over $100 \mathrm{~nm}$ can cause MTE increases as large as $30 \mathrm{meV}$. For larger accelerating electric fields, the MTE reduces with increasing accelerating field. For smaller accelerating electric fields, the MTE remains constant due to the influence of the varying surface potential.

Real photocathode surfaces can exhibit both physical and chemical roughness simultaneously. The physical roughness tends to increase the MTE with increasing accelerating gradient whereas the effect of chemical roughness on MTE diminishes with increasing accelerating gradient. Hence predicting the combined effect of both on the MTE as a function of accelerating gradient is non-trivial.

In this paper, based on Gorlov's method of calculating the electric potential, we first develop a technique to calculate the electric potential and fields close to a surface exhibiting realistic physical and chemical roughness. The method can use AFM and KPFM measurements of real photocathode surfaces as inputs to calculate the electric fields close to the surface for any given accelerating gradient. As a demonstration, we calculate the electric fields close to the surface of an alkali-antimonide cathode measured using AFM and KPFM. Then, we numerically calculate the trajectories of electrons in these fields and calculate the expected MTE variation with the accelerating gradient due to the combined effect of physical and chemical roughness. We show that for a real alkaliantimonide surface the combined effect of physical and chemical roughness would initially cause the MTE to reduce with increasing electric field, go to a minimum and then increase again. All computation work has been conducted using MATLAB $^{\circledR}$ [22].

\section{CALCULATION OF ELECTRIC FIELDS CLOSE TO THE SURFACE}

\section{A. Description of method}

To track a single electron through space close to the cathode surface, we need to have an accurate model of the electric field at all positions. In principle, any finite element or boundary element method can work for this calculation, however due to the small scale of the surface roughness relative to the spatial scale of the problem, these methods become impractical owing to large computational requirements. To circumvent this issue, Gorlov suggested a formalism based on modeling the electric potential using a combination of sinusoidal and exponential functions and applied it to an equipotential surface with nanoscale physical roughness [18]. Here we extend Gorlov's formalism to 


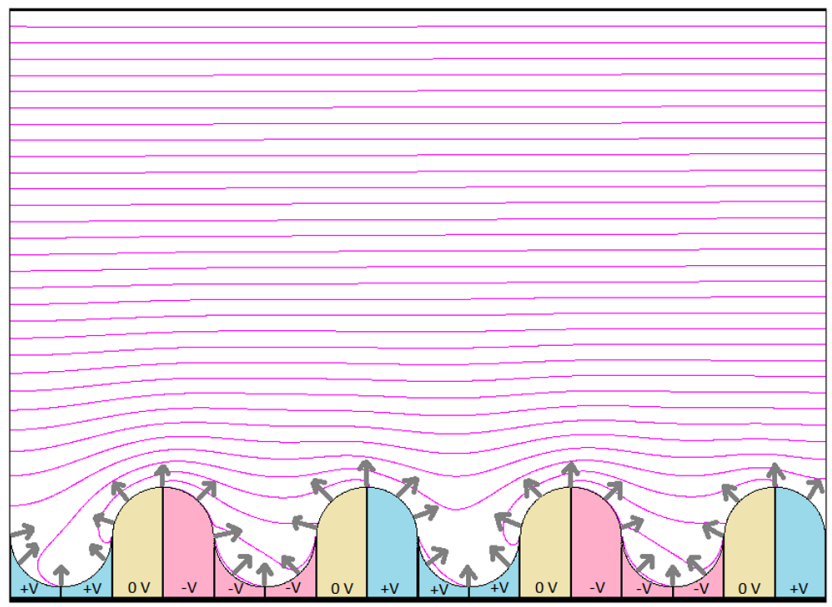

FIG. 1. Parallel-plate model to simulate equipotential lines produced by surface and chemical roughness. The gray arrows represent electrons emitted from the cathode. The blue regions show areas with positive surface potential, the beige regions show areas with zero surface potential and the red regions show areas with negative surface potentials. This image is only meant to describe the model used to solve the problem and a rough depiction of the equipotential lines close to a surface with physical and chemical roughness and does not depict a real cathode surface used for simulations in this paper.

model electric fields close to physically rough surfaces with varying surface potential.

We model this problem using a parallel-plate capacitor where one plate is the physically rough cathode with varying surface potential (generally obtained from an AFM and KPFM measurement) and the other plate is flat and held at a distance $L_{0}$ away from the cathode at a potential $\Phi$. Fig. 1 is a drawing that roughly sketches this model and the equipotential lines produced in between the plates. The distance $L_{0}$ is chosen such that it is much larger than the lateral $x, y$ dimensions of the measured surface and the maximum amplitude of the physical roughness. This model assumes that the cathode surface is infinite in the $x$ and $y$ directions with periodic images of the measured surface.

To calculate the electric potential between the two plates, let us assume that the lengths of the measured surface in the $x$ and $y$ directions are $L_{x}$ and $L_{y}$ respectively. Let us assume that the measured surface height is given by $f(x, y)$ and the measured surface potential is given by $U_{0}(x, y)$. Now the problem reduces to solving the Poisson's equation $\nabla^{2} U=0$ for the potential $U(x, y, z)$ under the following boundary conditions:

$$
\begin{gathered}
\left.U\right|_{z=L_{0}}=\phi \\
U\left(x+L_{x}, y+L_{y}\right)=U(x, y) \\
\left.U\right|_{z=f(x, y)}=U_{0}(x, y)
\end{gathered}
$$

One ansatz solution for $U(x, y, z)$ that satisfies the Poisson equation and the boundary conditions given by (1) and (2) is:

$$
\begin{aligned}
U= & \sum_{m_{1}, n_{1}=0}^{N} c_{m_{1}, n_{1}} K \cos \left(\frac{2 \pi}{L_{x}} m_{1} x\right) \cos \left(\frac{2 \pi}{L_{x}} n_{1} y\right) \\
& +\sum_{m_{2}, n_{2}=0}^{N} c_{m_{2}, n_{2}} K \cos \left(\frac{2 \pi}{L_{x}} m_{2} x\right) \sin \left(\frac{2 \pi}{L_{x}} n_{2} y\right) \\
& +\sum_{m_{3}, n_{3}=0}^{N} c_{m_{3}, n_{3}} K \sin \left(\frac{2 \pi}{L_{x}} m_{3} x\right) \cos \left(\frac{2 \pi}{L_{x}} n_{3} y\right) \\
& +\sum_{m_{4}, n_{4}=0}^{N} c_{m_{4}, n_{4}} K \sin \left(\frac{2 \pi}{L_{x}} m_{4} x\right) \sin \left(\frac{2 \pi}{L_{x}} n_{4} y\right)+\frac{\varphi z}{L_{0}}
\end{aligned}
$$

Where $K=\frac{e^{-\gamma z}-e^{\left(z-2 L_{0}\right) \gamma}}{1-e^{-2 L_{0} \gamma}}, \gamma=2 \pi \sqrt{\left(\frac{m}{L_{x}}\right)^{2}+\left(\frac{n}{L_{y}}\right)^{2}}$. By using the assumption that the anode distance $L_{0} \gg \max [f(x, y)]$, $L_{x}$ and $L_{y}$, we reduce the exponential term of the coefficient $K$ to be $K \approx e^{-\gamma z}$. Equation (4) can be written in a more concise manner as:

$$
U=\sum_{n=1}^{4(N+1)^{2}} c_{i} \times \phi_{i}(x, y)
$$

where $c_{i}$ are the coefficients (either of $c_{m_{1}, n_{1}}, c_{m_{2}, n_{2}}, c_{m_{3}, n_{3}}$ or $\left.c_{m_{4}, n_{4}}\right)$ for the various corresponding basis functions $\phi_{i}$ (the four listed in Eq. (4)).

Now the problem reduces to finding the coefficients $c_{i}$ corresponding to the basis functions $\phi_{i}$ such that the boundary condition given by Eq. (3) is satisfied. Note that the basis functions $\phi_{i}$ are not orthonormal as they contain the exponential term $K$ in order to satisfy the Poisson equation. Here we use the Ritz method [18] to find the set of coefficients $c_{i}$ that minimizes the difference between the measured surface potential $U_{0}(x, y)$ and the potential obtained from Eq. (5) over the surface. This difference $\varepsilon$ is given by:

$$
\varepsilon=\left(\iint_{S}(\xi)^{2} d x d y\right)^{1 / 2}
$$

where $S$ is the surface $y=0$ to $L_{y}$ and $x=0$ to $L_{x}$, and $\xi=U_{0}(x, y)-\sum_{i=1}^{4(N+1)^{2}} c_{i} \cdot \phi_{i}[x, y, f(x, y)]$. By differentiating $\varepsilon^{2}$ with respect to each $c_{i}$ coefficient and setting the derivatives to zero, we obtain series of $4(N+1)^{2}$ equations with the $c_{i}$ coefficients as unknowns. This series of equations can be expressed in the form of a matrix equation as:

$$
\vec{B}=\mathbf{A} \cdot \vec{C}
$$

where $\vec{B}$ is a vector of length $4(N+1)^{2}$ with elements given by: 


$$
b_{j}=\iint_{S} U_{0}(x, y) \cdot \phi_{j}(x, y) d x d y
$$

A is a $4(N+1)^{2} \times 4(N+1)^{2}$ matrix with elements given by

$$
a_{i j}=\iint_{S}\left(\phi_{i}(x, y) \cdot \phi_{j}(x, y) d x d y\right.
$$

and $\vec{C}$ is a vector with elements $c_{i}$. The vector $\vec{C}$ can then be calculated by inverting the $\boldsymbol{A}$ matrix and is given by $\vec{C}=\mathbf{A}^{-1} \vec{B}$. Once the $c_{i}$ coefficients are known, $U(x, y, z)$ can be analytically computed from Eq. (5). The electric field can also be calculated by differentiating the potential $U$. If the basis functions were orthonormal, A would be exactly the identity matrix and $\vec{B}$ would be equal to $\vec{C}$, resulting in a standard Fourier transformation.

\section{VALIDATION}

We verify the validity of the above formalism by comparing its results to those obtained from various sources with well-defined potentials. First we compare the results obtained by the above formalism to those obtained by Bradley [15] for an equipotential surface with a 1-D sinusoidal variation. Bradley analytically calculated the transverse $(x)$ and longitudinal $(z)$ fields for an equipotential surface given by $z=a \cdot \cos (p x)$, placed in an electric field of strength $E_{0}$ to first order under the assumption $a \ll p$. The electric fields are as follows:

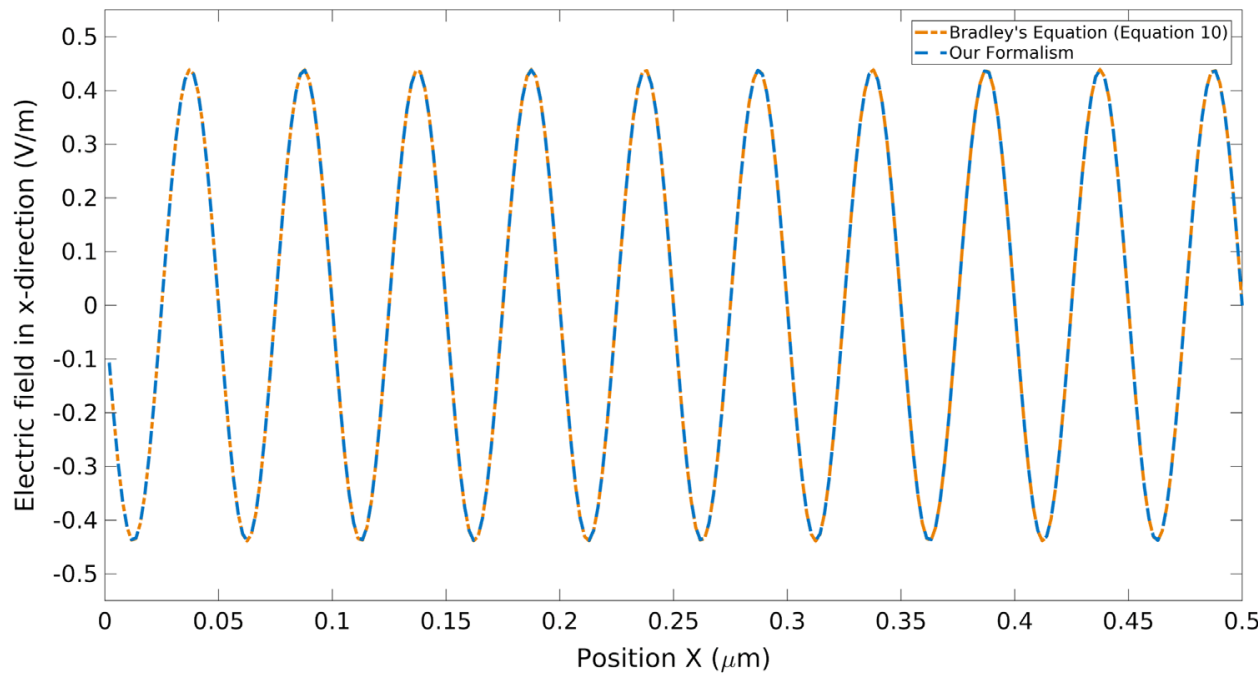

(a) Electric field in $\mathrm{x}$-direection

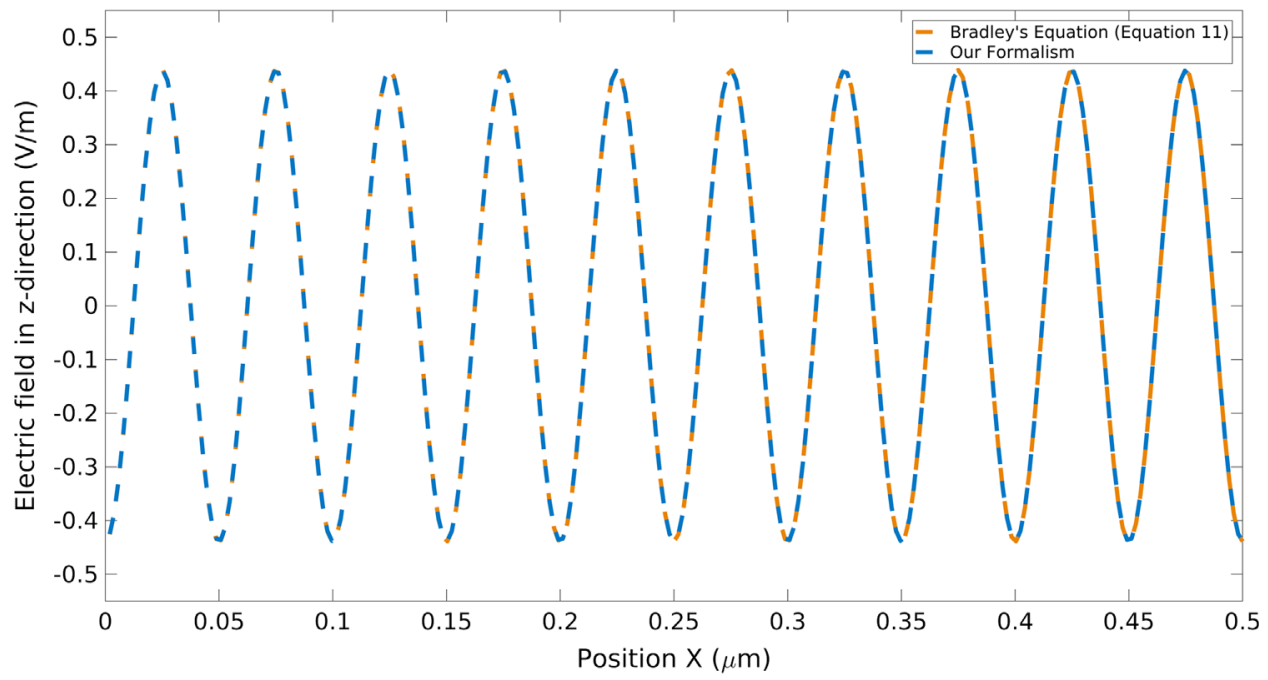

(b) Electric field in z-direction

FIG. 2. Comparison of electric fields in $x$ direction (a) and in $z$ direction (b) $100 \mathrm{~nm}$ away from a 1-D sinusoidal surface as calculated using Bradleys equations (orange dashes) and using the formalism presented here (blue dashes). The z-direction is plotted with the constant electric fields removed. The two match to within three significant digits. 


$$
\begin{aligned}
& E_{x}=E_{0} a p \cdot e^{-p z} \cdot \sin (p x) \\
& E_{z}=E_{0}+E_{0} a p \cdot e^{-p z} \cdot \cos (p x)
\end{aligned}
$$

In Fig. 2 we compare the electric fields computed by our formalism against Bradley's formulas. Using inputs of $E_{0}=1.0 \mathrm{MV} / \mathrm{m}, a=1.0 \mathrm{~nm}$, and $p=2 \pi / 50 \mathrm{~nm}^{-1}$, we modeled the electric fields at the source surface. For this source, the simulated surface size was $500 \times 500 \mathrm{~nm}$ sampled on a equispaced mesh of size $256 \times 256$. The surface is assumed to be equipotential. The fields are evaluated on the plane $z=100 \mathrm{~nm}$ above the cathode surface. The distance was chosen arbitrarily to verify the accuracy of the model near the surface.

As seen in Fig. 2, our formalism produces electric fields identical to those obtained from Bradley's equations with an accuracy down to three significant digits. The rms difference of the electric fields were $5.67 \times 10^{-4} \mathrm{~V} / \mathrm{m}$ for both the $x$-direction and $z$-direction. The small phase discrepancy in the results comes from the lack of higher order terms in Bradley's solution.

To check the validity of this method for a surface with varying surface potential, we utilized electric potential solutions published by Karkare and Bazarov [21] to examine a surface with constant height but sinusoidally varying surface potential. Using the same general parameters as before, we set the surface height to be constant and vary the surface potential as $U_{0}(x, y)=h \sin \left(p_{X} x\right) \cdot \sin \left(p_{y} y\right)$. For such a surface placed in an external electric field of strength $E_{0}$ the potential near the surface is analytically given by:

$$
U(x, y, z)=E_{0} z+h e^{-\gamma z} \cdot \sin \left(p_{x} x\right) \cdot \sin \left(p_{y} y\right)
$$

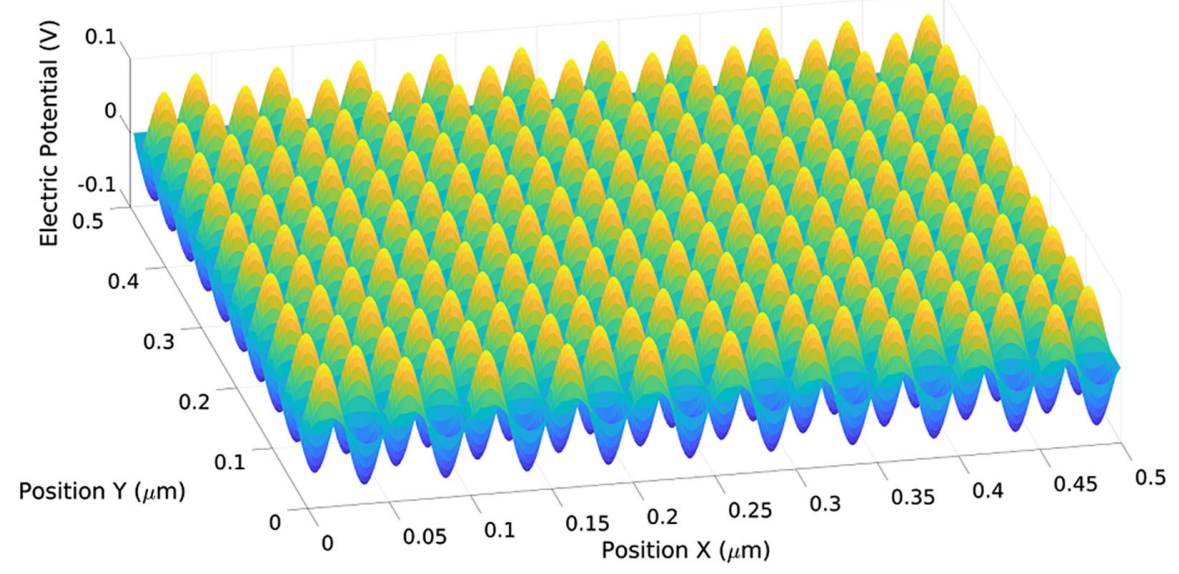

(a) Electric potential from Eq. (12)

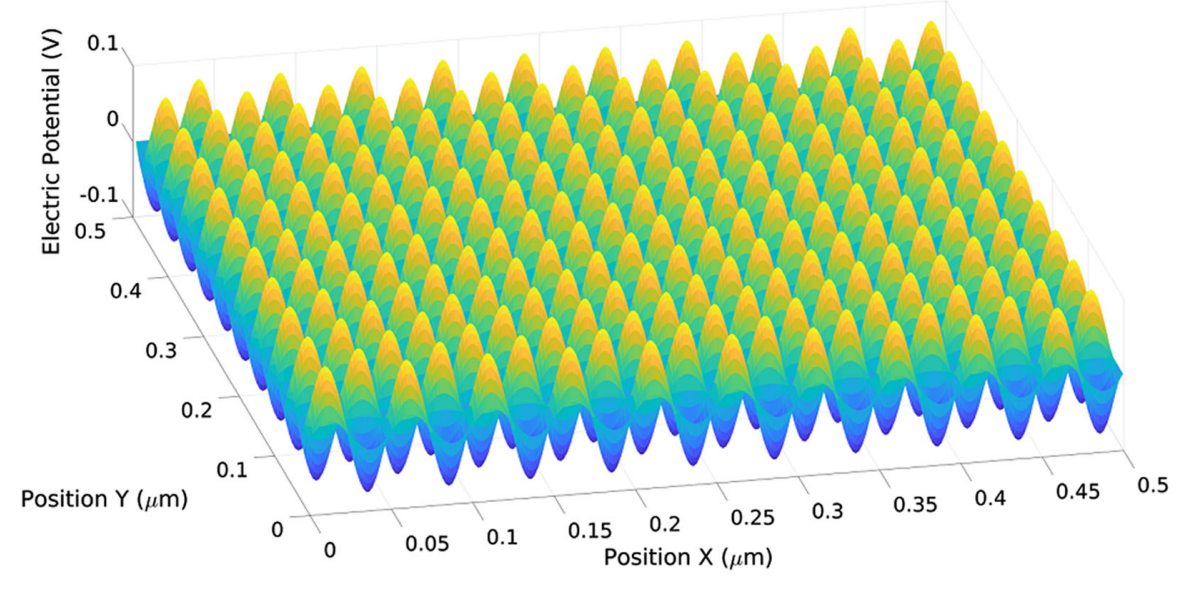

(b) Electric potential from our formalism

FIG. 3. Comparison of the electric potential computed $10 \mathrm{~nm}$ above a uniform cathode surface with $2 \mathrm{D}$ sinusoidally varying surface potential using (a) Eq. (12) as obtained from [21] and (b) using the formalism presented in this paper. The offset term of the electric potential is subtracted to show the variation. The two match to within $1.6 \%$. 
where $E_{0}$ is the applied external electric field, $h$ is the amplitude of the surface potential variation and $\gamma=\sqrt{p_{x}^{2}+p_{y}^{2}}$. As with the previous case, we set the applied electric field as $1.0 \mathrm{MV} / \mathrm{m}$. We used a surface potential amplitude $h=0.5 \mathrm{~V}$, and frequencies $p_{x}=$ $p_{y}=2 \pi / 50 \mathrm{~nm}^{-1}$. Using the another arbitrary distance close to the surface, the plots in Fig. 3 compare the potential $U$ at $z=10 \mathrm{~nm}$ above the surface when computed using Eq. (12) and when computed via our formalism. The rms difference of the calculated using the two methods is $0.0014 \mathrm{~V}$ which is about $1.6 \%$.

It can be seen that our formulation, from Eq. (5), reduces to Bradley's and Bazarov's results for the surface variations that they have considered respectively. This formulation provides a more general result that can be applied to a surface with any physical and potential variation and thus will be very useful to investigate real cathode surfaces.

\section{MTE CALCULATION FOR ALKALI-ANTIMONIDE SURFACE}

$\mathrm{A} \mathrm{Cs}_{3} \mathrm{Sb}$ cathode was grown in a UHV growth chamber using the co-deposition technique. The details of the growth are given elsewhere [19]. The cathode was then transferred into an atomic force microscope (AFM) with the Kelvin probe force microscopy (KPFM) capability [23] under UHV conditions. Surface height and potential maps were measured for this cathode surface using the AFM and KPFM techniques respectively. The measured surface map is shown in Fig. 4(a). Note that these measurements are only preliminary and are used here as an example of a physically and chemically rough surface. Detailed results from these measurements will be published elsewhere.

The electric potential of the surface is reproduced and plotted on the surface in Fig. 4(b) using our formalism. For this surface we obtain an rms error $\epsilon=9.96 \mathrm{mV}$, which

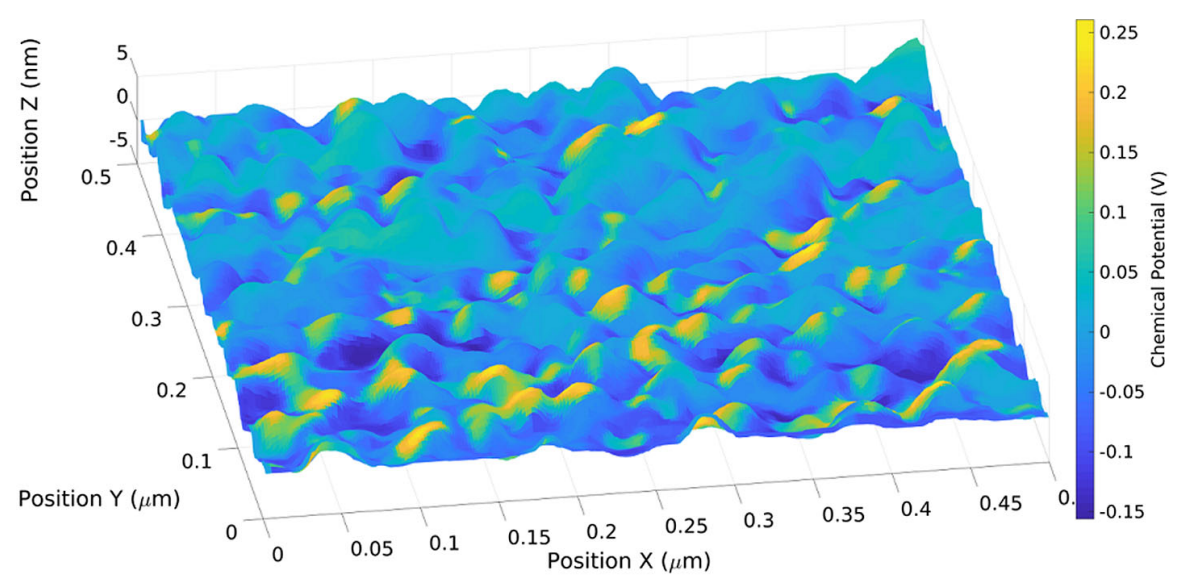

(a) AFM with KPFM surface potential

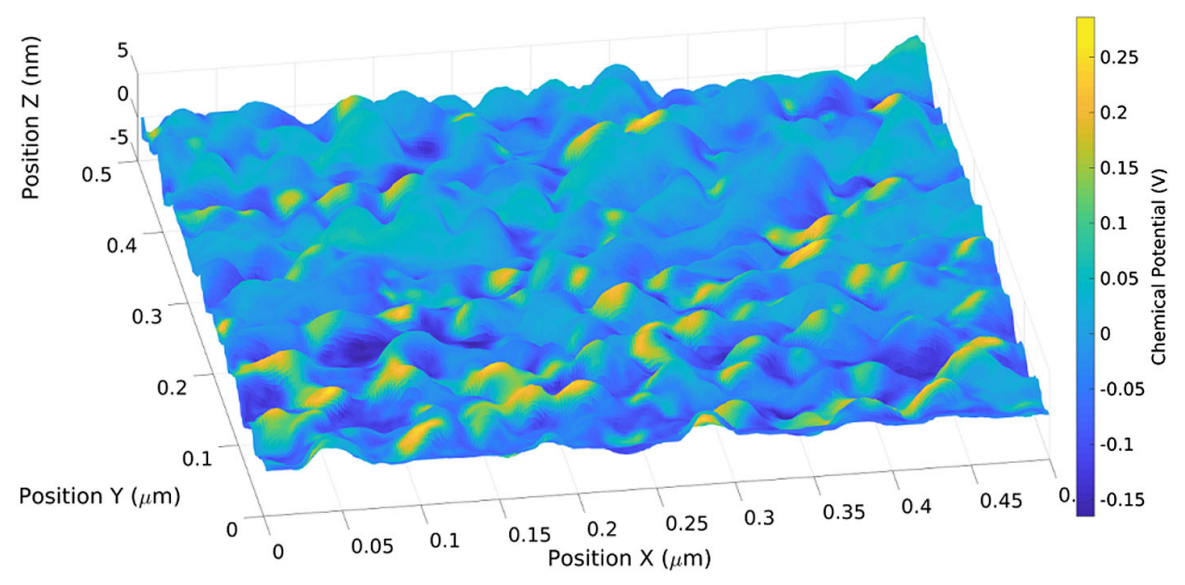

(b) AFM with computed surface potential

FIG. 4. (a) Surface physical and chemical roughness of $\mathrm{Cs}_{3} \mathrm{Sb}$ as measured by AFM and KPFM. (b) Surface potential as calculated by our formalism on the rough surface. The color coding denotes the potential variation on the surface. 
is a fractional error of $2.2 \%$. The rms error depends upon the sampling rate, sample size of the surface $\left(L_{x}, L_{y}\right)$, the number of frequencies $N$ used in our formulation, the periodicities of surface roughness, and the amplitudes of surface height and potential variation. The larger is the number of frequencies used, the smaller is the rms error. However, this formalism becomes numerically unstable for very large frequencies [18].

Utilizing the derived electric fields computed using the above formalism for the measured surface height and potential maps, we simulated the trajectory of electrons as they would be accelerated away from such a cathode surface placed in an accelerating electric field. The electrons were tracked until the effects of the surface height and potential variations become negligible. We use a symplectic Velocity Verlet [24] tracker for this purpose. Electrons were launched from a uniform $100 \times 100(x, y)$ grid on the surface with zero kinetic energy. The number of frequencies used to calculate the electric fields $(N)$ was set to 45 and the time increment for electron trajectory calculation was set to $5.0 \mathrm{fs}$. The time step was chosen to be small enough that doubling the time step did not change any of the electron trajectories significantly.

The MTE of these electrons was calculated as the average transverse kinetic energy of all the electrons after they are far enough from the surface that the electric fields due to the surface height and potential variations are negligible. This gives an estimate of the surface variation contribution to the MTE from a real cathode surface. The calculated MTE as a function of the accelerating electric field is shown in Fig. 5 (black solid line). Figure 5 also

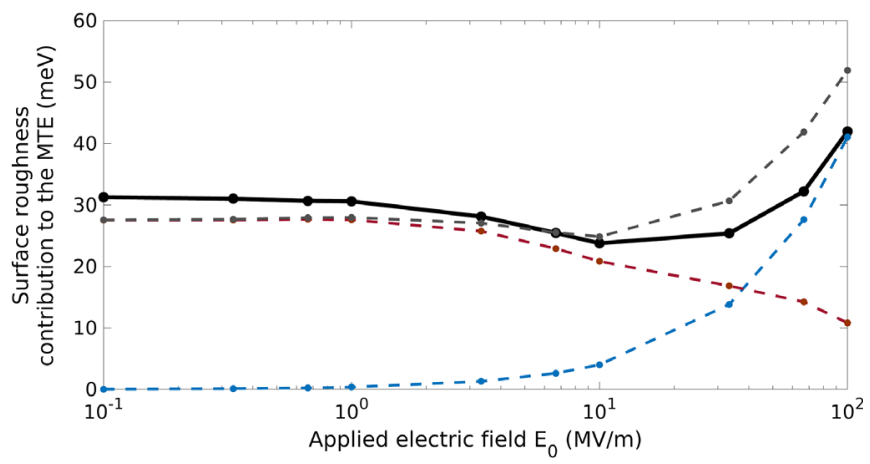

FIG. 5. Surface nonuniformities contribution to the MTE from the $\mathrm{Cs}_{3} \mathrm{Sb}$ photocathode surface shown in Fig. 4 (solid black line). At lower electric fields the MTE is dominated by the variations in surface potential, whereas, at higher electric fields it is dominated by the physical surface roughness. The figure also shows the MTE obtained from the surface assuming only potential variations and no height variations (red dotted line), assuming only height variations and a constant potential (blue dotted line) and the sum of the two (gray dotted line). Despite the same surface potential on the surfaces corresponding to the solid black line and the dashed gray/red line, the $3 \mathrm{meV}$ difference in MTE at zero electric field arises due to the fact that these surfaces have a different physical roughness and hence different surface electric fields despite the same surface potential. shows the MTE obtained from the surface assuming only potential variations and no height variations (red dotted line), assuming only height variations and a constant potential (blue dotted line) and the sum of the two (black dotted line). It is clear that a simple addition of the MTE obtained from a surface with only potential variation and a surface with only height variations is not equal to the MTE calculated from a surface which has both the potential and height variations simultaneously.

At smaller accelerating electric fields, the contribution of height variations is negligible, however the MTE is dominated by the surface potential variations. This contribution remains nearly invariant at a value of $\approx 31 \mathrm{meV}$ until the electric field exceeds $1 \mathrm{MV} / \mathrm{m}$ after which it reduces with increasing electric field. This reduction is expected because, as the accelerating field increases the electrons spend a smaller time in the region with significant transverse electric fields due to surface potential changes. The contribution of the surface height variation to the MTE is proportional to the accelerating electric field. Hence, as the accelerating electric field is increased further, the MTE reaches a minimum and starts to increase with the accelerating electric field.

We note here that Fig. 5 shows only the surface nonuniformities contribution to the MTE. In reality, several other factors like the cathode temperature, photon energy in excess of the work function and the electronic band-structure of the cathode materials also contribute significantly to the MTE. In principle, by cryocooling the cathode and by tuning the photon energy very close to the photoemission threshold, it should be possible to minimize the contributions of all these factors to a point where only the contribution of surface nonuniformities will be significant. The low field limit of MTE is significantly above the value for $k_{B} T$ at room temperature $(\approx 25 \mathrm{meV})$, but reduces to approximately this value at the optimum accelerating field, around $10 \mathrm{MV} / \mathrm{m}$. It should also be noted that various factors like nonuniform emission from the surface due to nonuniform work function and the initial kinetic energy distributions of the electrons have been ignored in this calculation. Including these effects will be a subject of future work.

The roughness contribution to the MTE calculated here at low electric fields is $32 \mathrm{meV}$. This is already much larger than the MTE of $22 \mathrm{meV}$ measured from co-deposited $\mathrm{Cs}_{3} \mathrm{Sb}$ at $90 \mathrm{~K}$ temperature at the photoemission threshold [9]. One reason for this discrepancy could be that the surface of the cathode used to measure the MTE was significantly smoother than the surface shown in Fig. 4. This discrepancy underscores a need for complete surface characterization along with MTE measurements on the same cathode surface.

\section{CONCLUSION AND FUTURE WORK}

Advancements in codeposition methods of alkaliantimonide thin films have reduced the physical roughness and the extent of local changes in chemical potential 
significantly. Today, alkali-antimonide photocathodes with height variations of a few $\mathrm{nm}$ and chemical potential amplitudes of $0.05 \mathrm{~V}$ over length scales of 50-200 nm [11] can be grown using these co-deposition methods. Such variations can, in principle, be measured accurately using AFM and KPFM. We developed a MATLAB ${ }^{\circledR}$ [22] based program which allows us to calculate a lower bound to the MTE due to such surface variations at various accelerating electric fields. This knowledge is essential to understand the effects of cathode surface variations on MTE and develop photocathodes that minimize these effects to obtain MTE well below the room temperature thermal limit.

We showed how the electric fields close to any rough surface with varying surface potential can be calculated accurately using a spectral expansion. We verified the computational accuracy of the formalism using simulated surfaces with known analytic solutions to the electric fields. Then we calculated the contributions of the surface (physical and chemical) nonuniformities by launching electrons from the surface and numerically tracing their trajectories in the calculated electric fields close to the surface. We showed that for a codeposited alkali-antimonide surface, the surface potential variations can limit the MTE at low electric fields to around $30 \mathrm{meV}$. At higher electric fields the contributions of the surface potential reduce and the MTE goes to a minimum. At even higher electric fields, the effects of physical surface roughness dominate the MTE increasing it again.

In this work, we assume that the electrons have no initial kinetic energy and "float" away from the surface under the influence of the applied and surface electric fields. This is partly valid if the photon energy is less than the work function, and the photocathode is cryogenically cooled to liquid helium temperatures. Then the initial velocity of each electron would be near zero, but would vary due to the chemical potential. The number of higher energy electrons would reduce exponentially with the tail of the Fermi distribution. In such a scenario, we can reasonably assume that all electrons are emitted with zero kinetic energy, and weighting the electrons by a factor of $e^{-\frac{U_{0}}{k T}}$, where $U_{0}$ is the surface chemical potential, can be sufficient.

A more accurate way to incorporate the effects of surface and chemical roughness on the number and initial energy of electrons would be to emit electrons with distributions of energies, at distributions of angles, from each point respectively. The energy distributions would depend on the photon energy and the local surface potential. One feasible way to address this is to use a Monte-Carlo based approach to sample the energy and angular space. The hope is that even a dilute sampling of electron energies and angles from each spatial point would result in a statistically accurate MTE when sampled over the entire surface. Further work on this program should work to remove the initial zero kinetic energy and the uniform electron emission assumptions.
These developments on the program would allow us to characterize the statistical nature of the emission in the far field. If we have a range of surface potentials close to the average threshold, then only the areas of low chemical potential will emit. The result will be an uneven far field distribution of electrons; and such speckling in the positional distribution would have an effect on the evolution of space charge. The aftermath would not be correlated space charge; it would represent an uncorrectable space charge limited MTE, and the program in its current form will not calculate these effects. However, we can use this program give us some idea of the speckle introduced in the electron beam due to surface nonuniformities. This speckle pattern can then be used as an input to other beam dynamics codes that allow the calculation of space charge effects.

\section{ACKNOWLEDGMENTS}

This work was supported by the Director, Office of Science, Office of Basic Energy Sciences of the U.S. Department of Energy, under Contracts No. KC0407ALSJNT-I0013 and No. DE-AC02-05CH11231, and by the National Science Foundation under Grant No. Phy1549132, the Center for Bright Beams.

[1] P. Emma, R. Akre, J. Arthur, R. Bionta, C. Bostedt, J. Bozek, A. Brachmann, P. Bucksbaum, R. Coffee, F.-J. Decker et al., First lasing and operation of an angstromwavelength free-electron laser, Nat. Photonics 4, 641 (2010).

[2] A. H. Zewail, 4d ultrafast electron diffraction, crystallography, and microscopy, Annu. Rev. Phys. Chem. 57, 65 (2006).

[3] P. Musumeci, J. G. Navarro, J. B. Rosenzweig, L. Cultrera, I. V. Bazarov, J. Maxson, S. Karkare, and H. A. Padmore, Advances in bright electron sources, Nucl. Instrum. Methods Phys. Res., Sect. A, DOI: 10.1016/j.nima.2018.03.019 (2018).

[4] D. Dowell and J. Schmerge, Quantum efficiency and thermal emittance of metal photocathodes, Phys. Rev. Accel. Beams 12, 074201 (2009).

[5] J. Feng, J. Nasiatka, W. Wan, S. Karkare, J. Smedley, and H. A. Padmore, Thermal limit to the intrinsic emittance from metal photocathodes, Appl. Phys. Lett. 107, 134101 (2015).

[6] J. Maxson, P. Musumeci, L. Cultrera, S. Karkare, and H. A. Padmore, Ultrafast laser pulse heating of metallic photocathodes and its contribution to intrinsic emittance, Nucl. Instrum. Methods Phys. Res., Sect. A 865, 99 (2017).

[7] S. Karkare, D. Dimitrov, W. Schaff, L. Cultrera, A. Bartnik, X. Liu, E. Sawyer, T. Esposito, and I. V. Bazarov, Monte carlo charge transport and photoemission from negative electron affinity GaAs photocathodes, J. Appl. Phys 113, 104904 (2013).

[8] K. L. Jensen, Scattering and the relationship between quantum efficiency and emittance, J. Appl. Phys. 113, 056101 (2013). 
[9] L. Cultrera, S. Karkare, H. Lee, X. Liu, I. V. Bazarov, and B. Dunham, Cold electron beams from cryocooled, alkali antimonide photocathodes, Phys. Rev. Accel. Beams 18, 113401 (2015).

[10] J. Xie, M. Demarteau, R Wagner, S. Schubert, M. Gaowei, K. Attenkofer, J. Walsh, J. Smedley, J. Wong, J. Feng, H. A. Padmore et al., Synchrotron x-ray study of a low roughness and high efficiency $K_{2} C s S b$ photocathode during film growth, J. Appl. Phys. 50, 205303 (2017).

[11] S. Karkare, S. Emamian, G. Gevorkyan, H. A. Padmore, and A. Schmidt, Physical and chemical roughness of alkaliantimonide cathodes, Proceedings of IPAC'18 (2018), p. 4259, http://ipac2018.vrws.de/papers/thpmf080.pdf.

[12] R. U. Martinelli, Effects of cathode bumpiness on the spatial resolution of proximity focused image tubes, Appl. Opt. 12, 1841 (1973).

[13] M. Krasilnikov, Impact of the cathode roughness on the emittance of an electron beam, Proc. FEL Conf. (2006), p. 583.

[14] K. L. Jensen, D. A. Shiffler, J. J. Petillo, Z. Pan, and J. W. Luginsland, Emittance, surface structure, and electron emission, Phys. Rev. Accel. Beams 17, 043402 (2014).

[15] D. J. Bradley, M. B. Allenson, and B. R. Holeman, The transverse energy of electrons emitted from GaAs photocathodes, J. Phys. D 10, 111 (1977).

[16] D. Xiang, W. Huang, Y. Du, L. Yan, R. Li, C. Tang, and Y. Lin, First principle measurements of thermal emittance for copper and magnesium, in Proceedings of the 22nd Particle Accelerator Conference, PAC-2007, Albuquerque, NM (IEEE, New York, 2007), p. 1049.

[17] Z. Zhang and C. Tang, Analytical study on emittance growth caused by roughness of a metallic photocathode, Phys. Rev. Accel. Beams 18, 053401 (2015).
[18] T. Gorlov, High-precision calculation of quasistatic field near a photocathode surface microrelief, J. Electrost. 65, 735 (2007).

[19] J. Feng, S. Karkare, J. Nasiatka, S. Schubert, J. Smedley, and H. A. Padmore, Near atomically smooth alkali antimonide photocathode thin films, J. Appl. Phys. 121, 044904 (2017).

[20] S. Karkare and I. V. Bazarov, Effect of nanoscale surface roughness on transverse energy spread from GaAs photocathodes, Appl. Phys. Lett. 98, 094104 (2011).

[21] S. Karkare and I. V. Bazarov, Effects of surface nonuniformities on the mean transverse energy from photocathodes, Phys. Rev. Applied 4, 024015 (2015).

[22] The Mathworks, Inc., MATLAB ${ }^{\circledR}$ Release 2018a, Natick, Massachusetts, United States.

[23] W. Melitz, J. Shena, A. C. Kummela, and S. Lee, Kelvin probe force microscopy and its application, Surf. Sci. Rep. 66, 1 (2011).

[24] W. C. Swope, H. C. Andersen, P. H. Berens, and K. R. Wilson, A computer simulation method for the calculation of equilibrium constants for the formation of physical clusters of molecules: Application to small water clusters, J. Chem. Phys. 76, 637 (1982).

Correction: The fifth sentence of the third paragraph in Sec. V contained an error in wording and has been fixed. The title in Ref. [9] was inaccurate and has been resolved.

Second Correction: The name of the first author was missing a second initial and has been modified. 